\title{
Lack of ClC-2 Alleviates High Fat Diet-Induced Insulin Resistance and Non-Alcoholic Fatty Liver Disease
}

\author{
Dongxia Fua Haibin Cui ${ }^{\mathrm{a}} \quad$ Yunna Zhang ${ }^{\mathrm{a}}$ \\ ${ }^{a} 2^{\text {nd }}$ Department of Endocrinology, ${ }^{\mathrm{b}} 1^{\text {st }}$ Department of Oncological Surgery, Cangzhou Central Hospital, \\ Hebei, People's Republic of China
}

\section{Key Words}

Non-alcoholic fatty liver disease - Obesity • Lipid accumulation • Insulin resistance - IRS-1/ Akt/mTOR signaling $\bullet \mathrm{ClC}-2$

\begin{abstract}
Background/Aims: Non-alcoholic fatty liver disease (NAFLD) is the most common cause of chronic liver disease. This study aims to investigate whether chloride channel 2 (ClC2) is involved in high fat diet (HFD)-induced NAFLD and possible molecular mechanisms. Methods: CIC-2 expression was liver-specifically downregulated using adeno-associated virus in C57BL/6 mice treated with a chow diet or HFD for 12 weeks. Peripheral blood and liver tissues were collected for biochemical and pathological estimation respectively. Western blotting was applied to detect the protein expressions of lipid synthesis-related enzymes and the phosphorylated level of IRS-1, Akt and mTOR. Results: CIC-2 mRNA level was significantly increased in patients with non-alcoholic steatohepatitis, which positively correlated with the plasma levels of alanine transaminase (ALT), aspartate transaminase (AST) and insulin. Knockdown of $\mathrm{ClC}-2$ in liver attenuated HFD-induced weight gain, obesity, hepatocellular ballooning, and liver lipid accumulation and fibrosis, accompanied by reduced plasma free fatty acid (FFA), triglyceride (TG), total cholesterol (TC), ALT, AST, glucose and insulin levels and homeostasis model of insulin resistance (HOMA-IR) value. Moreover, HFD-treated mice lacking $\mathrm{ClC}-2$ showed inhibited hepatic lipid accumulation via regulating lipid metabolism through decreasing sterol regulatory element binding protein (SREBP)-1c expression and its downstream targeting enzymes such as fatty acid synthase (FAS), HMG-CoA reductase (HMGCR) and acetyl-Coenzyme A carboxylase (ACC $\alpha$ ). In addition, in vivo and in vitro results demonstrated that $\mathrm{ClC}-2$ downregulation in HFD-treated mice or HepG2 cells increased the sensitivity to insulin via activation of IRS-1/Akt/mTOR signaling pathway. Conclusion: Our present study reveals a critical role of $\mathrm{ClC}-2$ in regulating metabolic diseases. Mice lacking $\mathrm{CIC}-2$ are associated with a remarkably beneficial metabolic phenotype, suggesting that decreasing $\mathrm{ClC}-2$ may be an attractive therapeutic strategy for the treatment of NAFLD.
\end{abstract}




\section{Cellular Physiology Cell Physiol Biochem 2018;45:2187-2198 \begin{tabular}{l|l} 
and Biochemistry Published online: March 15, 2018 & $\begin{array}{l}\text { (c) } 2018 \text { The Author(s). Published by S. Karger AG, Basel } \\
\text { www.karger.com/cpb }\end{array}$
\end{tabular}}

Fu et al.: CIC2 Deficiency Ameliorates Non-Alcoholic Fatty Liver Disease

\section{Introduction}

Non-alcoholic fatty liver disease (NAFLD) is considered as the hepatic component of metabolic syndrome not due to excess alcohol consumption, and is the most common cause of chronic liver disease in the world [1]. NAFLD encompasses a spectrum of disorders ranging from simple hepatic steatosis, which is characterized by lipid accumulation in hepatocytes, to non-alcoholic steatohepatitis (NASH), which can further develop to cirrhosis and hepatocellular carcinoma [2,3]. Moreover, NAFLD and NASH confer an increased risk of cardiovascular diseases [4]. Despite many factor including oxidative stress, fatty acid syntheses and inflammation have been suggested to be implicated in NAFLD [5], the precise mechanism of the onset and development of NAFLD remains to be clearly defined.

The prevailing theory of NAFLD pathogenesis is commonly based on the 'double-hit' hypothesis [6]. Insulin resistance (IR) and abnormal hepatocellular lipid accumulation represents the 'first hit', which in turn trigger "second hit" such oxidative stress, inflammation, apoptosis and fibrosis, thus leading to the occurrence of NASH. Therefore, targeting IR and lipid dysregulation that occurs with progressive NAFLD will be extremely important for developing more sophisticated treatment options for NAFLD patients. IR, the pivotal mechanism in primary NAFLD, is defined as decreased sensitivity to insulin and associated with defective insulin signaling transduction, uptake of fatty acids by the liver, and synthesis of triglycerides (TG) [7].

Chloride ions $\left(\mathrm{Cl}^{-}\right)$are common and abundant anions in organism, regulating a variety of physiological and pathological processes [8]. Increasing lines of evidences have suggested that dysregulated intracellular $\mathrm{Cl}^{-}$level is associated with vascular inflammation, hypertension, lipid accumulation and atherosclerosis [9-11]. Chloride channels (ClCs) function as a kind of permeable channel or protein, which are encoded and regulated by the genes of $\mathrm{ClC}$ family [12]. ClC-2, a member of ClCs family, is almost ubiquitously expressed, including in the liver $[12,13]$. Recently, ClC-2 attracted increasing attention because $\mathrm{ClC}-2$ has been implicated in several diseases, such as cystic fibrosis, leukemia and diabetes mellitus $[12,14]$. Importantly, fatty acids can activate human $\mathrm{ClC}-2 \mathrm{Cl}^{-}$channel [15], further supports the involvement in metabolic disorders. However, the expression pattern and functional role of ClC-2 in NAFLD remain unknown. The aim of this study was to investigate the effect of ClC-2 on NAFLD and examine the underlying mechanisms.

\section{Materials and Methods}

\section{Materials}

Antibodies targeting sterol regulatory element binding protein (SREBP)-1c, fatty acid synthase (FAS), HMG-CoA reductase (HMGCR) and acetyl-Coenzyme A carboxylase (ACC $\alpha$ ) were purchased from Biosynthesis Biotechnology (Beijing, China). P-Akt (Ser473), Akt, p-mTOR (Ser2448), mTOR and $\beta$-actin antibodies were from Cell Signaling Technology (CA, USA). ClC-2, p-insulin receptor substrates 1 (p-IRS-1) (Tyr608/612), p-IRS-1 (Ser307) and IRS-1 were obtained from Abcam (MA, USA). SuperScript III FirstStrand Synthesis system, Dulbecco's modified Eagle's medium (DMEM), fetal calf serum (FBS), penicillin, streptomycin and lipofectamine 2000 reagent were obtained from Invitrogen (CA, USA).

\section{Human studies}

In this study, 14 subjects without NASH (near-normal liver histology, NNLH) and 31 NASH patients were recruited, which was approved by the Cangzhou Central Hospital Human Research Ethics Committee. Informed consent was obtained from each subject and all protocols were carried out in accordance with the guidelines of National Health and Medical Research Council of China. All subjects were received routine clinical assessment, including radiologic, hematologic, biochemical, and serologic testing. Male patients with daily alcohol consumption of $>30 \mathrm{~g}$ and female patients $>20 \mathrm{~g}$ were ruled out. Under laparoscopic guidance, a percutaneous core liver biopsy was performed with a 15-gauge biopsy device. Plasma samples were collected following an overnight fast on the day of liver biopsy. Patients were considered as NASH 


\section{Cellular Physiology Cell Physiol Biochem 2018;45:2187-2198

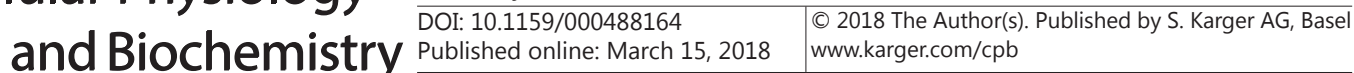 \\ Fu et al.: CIC2 Deficiency Ameliorates Non-Alcoholic Fatty Liver Disease}

if they had histopathological evidence of NASH, accompanied with abnormal liver enzymes or radiologic evidence of a fatty liver. NNLH was suspected in those patients with mildly elevated plasma liver enzymes, minimal histological changes or mild cholestasis.

\section{Animals}

Animal study was approved by the Institutional Animal Care and Use Committee of Cangzhou Central Hospital and carried out in accordance with the Guide for the Care and Use of Laboratory Animals of Cangzhou Central Hospital. 90 male C57BL/6 mice were purchased from the Jackson Laboratory (ME, USA) and maintained on a $12 \mathrm{~h} / 12 \mathrm{~h}$ light-dark cycle with free access to water and to the diets according to their treatment. The mice were randomly divided into 4 groups as follows: chow diet with adeno-associated virus (AAV)-null ( $n=15)$, chow diet with AAV-sh-ClC-2 (n=15), high fat diet (HFD) with AAV-null $(n=30)$ and high fat diet with AAV-sh-ClC-2 ( $\mathrm{n}=30)$. To knockdown ClC-2 expression specifically in liver, a small hairpin RNA sequence targeting mouse ClC-2 was subcloned into a specialized AAV8 vector provided by Addgene (MA, USA). AAV8 viral particles carrying an empty vector (AAV-null) were used as controls. At 10 weeks of age, mice were injected intravenously with AAV-sh-ClC-2 or AAV-null at a dose of $1 \times 10^{11}$ genome copies per mouse and then received a chow diet or HFD (45\% kcal fat, D12451, Research Diets, Inc., NJ, USA) for 12 weeks.

\section{Blood measurement}

Plasma alanine aminotransferase (ALT), aspartate aminotransferase (AST), high-density lipoprotein cholesterol (HDL-cholesterol), low-density lipoprotein cholesterol (LDL-cholesterol) (all from Jian Cheng Biological Engineering Institute, Nanjing, China), TG (Cayman, MI, USA), total cholesterol (TC), free fatty acids (FFA) (Wako Diagnostics, VA, USA), glycerol (Sigma, MO, USA) and lactate (Roche Diagnostics, Mannheim, Germany) were determined by biochemical assays. Blood glucose and plasma insulin measurements were performed in $10 \mathrm{~h}$ overnight-fasted mice using OneTouch Ultra Test Strips (Lifescan, BC, Canada) and Insulin ELISA Kit (Chrystal Chem, IL, USA), respectively. The homeostasis model assessment of IR (HOMA-IR) index was calculated as fasting blood glucose $\times$ fasting insulin/22.5. Glucose tolerance test (GTT) was monitored in $10 \mathrm{~h}$ fasted mice after a 12-week HFD. Mice were intraperitoneally injected with $1.5 \mathrm{~g}$ glucose/kg body weight. Insulin tolerance test (ITT) was performed with non-fasted mice after intraperitoneal injection of insulin (0.5 IU/kg body weight).

\section{Determination of biochemistry in liver tissue}

Mouse livers were harvested at the end of the experimental period. Liver homogenates were prepared in a 10 -fold volume $(\mathrm{v} / \mathrm{w})$ anhydrous alcohol, followed by centrifugation at $15000 \mathrm{rpm}, 4^{\circ} \mathrm{C}$ for $15 \mathrm{~min}$. The supernatant was collected for TC and TG determination using the same method in plasma measurement. Protein concentrations of liver tissue were quantified using the Enhanced BCA Protein Assay Kit (Beyotime, Jiangsu, China). Hepatic TC and TG were normalized to total proteins of tissue homogenates.

\section{QRT-PCR}

Total RNA (1 $\mu \mathrm{g}$ ) from human liver tissues was isolated using the RNeasy Mini kit (Qiagen, CA, USA) and was reverse-transcribed using the SuperScript III First-Strand Synthesis system. QRTPCR was performed using Fast SYBR ${ }^{\circledR}$ Green Master Mix Kit (Applied Biosystems, CA, USA) with an ABI Prism 7300 Fast Real-Time PCR system (Applied Biosystems). The ClC-2 mRNA expression was normalized to the mRNA levels of GAPDH. The specific primer sequences used for the amplification were as follows: ClC-2, 5'-CACAGGTGGTGGCATTGTTG-3' and 5'-GGACTTTCACACCCTGTGCT-3'; GAPDH, 5'-GGGCACGAAGGCTCATCATT-3' and 5'-AGAAGGCTGGGGCTCATTTG-3'.

\section{Western blotting}

Liver tissues or HepG2 cells were washed with cold phosphate buffered solution 3 times and lysed in RIPA buffer (Beyotime) containing 1\% protease inhibitor cocktail (Roche). After determination of protein concentrations, equal protein were separated on $8 \%$ or $10 \%$ Tris-HCL polyacrylamide gel and transferred to polyvinylidene difluoride (PVDF) membranes (Millipore, MA, USA). Membranes were blocked by $5 \%$ nonfat milk for $1 \mathrm{~h}$, followed by incubation with primary antibodies at $4{ }^{\circ} \mathrm{C}$ overnight. At the end of incubation period, the membranes were incubated with the secondary peroxidase-conjugated antibodies (goat anti- 


\section{Cellular Physiology Cell Physiol Biochem 2018;45:2187-2198 \begin{tabular}{l|l} 
and Biochemistry Published online: March 15, 2018 & $\begin{array}{l}\text { (c) } 2018 \text { The Author(s). Published by S. Karger AG, Basel } \\
\text { www.karger.com/cpb }\end{array}$
\end{tabular} \\ Fu et al.: CIC2 Deficiency Ameliorates Non-Alcoholic Fatty Liver Disease}

rabbit or rabbit anti-mouse, Beyotime) for $1 \mathrm{~h}$. Blots were detected by an enhanced chemiluminescence system (Pierce Biotech, IL, USA). The densitometry was quantified by ImageJ software (NIH, Maryland, USA).

\section{Body composition}

Mice were under 4\% chloral hydrate in anesthesia and analyzed for body mass composition through micro-computed tomography (micro-CT, INVEON, Siemens, TN, USA). Body mass composition was calculated using accompanied software.

\section{Histological examination}

The white adipose tissue (WAT), brown adipose tissue (BAT) and liver tissues were resected, dehydrated, infiltrated with paraplast embedding media (Sigma), fixed in 10\% formalin solution, embedded in fresh paraffin and subsequently sliced into 5 - $\mu$ m sections. Sections were stained with hematoxylin-eosin (HE), Oil Red 0 and masson trichrome. Images were captured using a Zeiss Axioplan2 fluorescence microscope (München, Germany). The mean diameter of adipocytes was calculated using the "Count/Size" function of Image-pro software (Media Cybernetics, MD, USA). All histological data were obtained by independent experimenters who were blinded to the physical outcome and other biological information of each sample.

\section{Metabolic cages}

Mice were individually housed in metabolic cages (Comprehensive Laboratory Animal Monitoring System, Columbus Instruments, $\mathrm{OH}, \mathrm{USA}$ ) for 24. Food and water intake was measured. The respiratory exchange ratio (RER) was calculated $\left(\mathrm{VCO}_{2} / \mathrm{VO}_{2}\right)$.

\section{Cell culture}

HepG2 cells were obtained from the American Type Culture Collection (ATCC, VA, USA) and cultured in DMEM supplemented with $10 \% \mathrm{FBS}$, antibiotics $\left(100 \mathrm{U} / \mathrm{mL}\right.$ penicillin and $100 \mathrm{mg} / \mathrm{mL}$ streptomycin at $37^{\circ} \mathrm{C}$ in a 95:5 air $/ \mathrm{CO}_{2}$ humidified atmosphere.

\section{CIC-2 siRNA transfection}

ThestealthsiRNAduplexoligoribonucleotidesagainsthumanClC-2(5'-CUACTACCATCATTCAUAACGU-3') was synthesized and purchased from FulenGen (Wuhan, China). HepG2 cells were transfected with ClC-2 siRNA or negative siRNA using lipofectamine2000 reagent for $48 \mathrm{~h}$ before insulin (Sigma) treatment.

\section{Adenovirus infection}

Human ClC-2 cDNA was obtained from Vigene Bioscience (Shandong, China) and cloned into pAdTrackCMV (Invitrogen). The recombinant plasmid was linearized by PacI digestion and then transfected into 293A cells with lipofectamine 2000 reagent to generate recombinant adenovirus. After amplification and purification, the titers of ClC-2 adenovirus (Ad-ClC-2) were assessed. An adenovirus bearing LacZ (Ad-Lacz, Clontech, CA, USA) was used a negative control. To make ClC-2 overexpression in HepG2 cells, cells were infected with Ad-ClC-2 or Ad-Lacz in FBS-free DMEM for 6 and then transferred into complete medium and cultured for $48 \mathrm{~h}$.

\section{Statistical analysis}

Data were expressed as mean \pm SEM. $n$ value represented the number of independent experiments or mouse. The correlation between ClC-2 expression and ALT, AST or insulin levels was examined by the Pearson correlation test. The statistical significance of differences between two groups was analyzed by Student t-test. Comparisons among multiple groups were performed by one-way ANOVA with post hoc Tukey's test. $\mathrm{P}<0.05$ was considered to be statistically significant.

\section{Results}

Increased ClC-2 expression correlated with plasma ALT, AST and insulin levels in NASH patients

Compared with NNLH individuals with mildly elevated plasma liver enzymes, patients with NASH significantly increased the value of body mass index and waist circumference 
and the plasma levels of ALT, AST, insulin, TG, TC and LDL-cholesterol, with reduced HDL-cholesterol (Table 1). Importantly, as shown in Fig. 1A, ClC-2 mRNA level was dramatically increased in NASH patients compared to NNLH patients. Furthermore, we analyzed the possible correlation between ClC-2 level and the above clinical and biochemical parameters. Interestingly, the results showed that the increased ClC-2 level was positively correlated with ALT, AST and insulin concentrations with the correlation coefficients of 0.6678 , 0.7057 and 0.6945 , respectively (Fig. 1B-D). These data suggest that ClC-2 level is reduced during liver injury and correlated with plasma ALT, AST and insulin levels.

Knockdown of CIC-2 inhibited HFD-induced obesity

Adiposity is the major feature of IR and NAFLD. To investigate whether ClC-2 is involved in NAFLDassociated obesity, 10-week old C57BL/6 mice were infected with liver specific AAV-sh-ClC-2 followed by a 12-week HFD. Consistent with increased ClC-2 level in NASH patient, ClC-2 expression in liver of HFD mice was also significantly increased. Injection of AAV-sh-ClC-2 inhibited the increased expression of ClC-2 (Fig. 2A). Although ClC-2 inhibition had no effect on body weight under chow diet treatment, mice lacking $\mathrm{ClC}-2$ in liver showed less weight gain after 3-12 weeks of the HFD treatment (Fig. 2B). AAVnull-treated HFD mice gained $13.7 \mathrm{~g}$ weight due to an increase of $13.1 \mathrm{~g}$ fat mass, while ClC-2 knockdown mice only gained $9.3 \mathrm{~g}$ weight due to an increase of $8.8 \mathrm{~g}$ fat mass. This supported that the reduction in body weight was associated a decrease of $4.3 \mathrm{~g}$ fat mass. No significant difference was observed in lean mass (Fig. 2C). Moreover, inguinal WAT (iWAT), gonadal WAT (gWAT) and interscapular BAT (iBAT) were all significantly reduced by $32.8 \%, 53.6 \%$ and $39.1 \%$, respectively, in AAV-sh-ClC-2 treated HFD mice when compared with AAV-null control (Fig. 2D). The morphological and histological changes in the adipose tissue were ameliorated in ClC-2 knockdown mice under HFD treatment, as evidenced by reduced adipocyte diameters in iWAT and iBAT (Fig. 2E-H). In addition, specific knockdown of ClC-2 in liver markedly inhibited HFD-induced the increase of plasma FFA, TG and TC levels, but produced no effect on glycerol and lactate levels (Fig. 2I-M).
Table 1. Clinical parameters according NNLH and NASH patients. Data were presented as mean \pm SEM. BMI, body mass index; ALT, alanine aminotransferase; AST, aspartate aminotransferase; HDL-c, high-density lipoprotein cholesterol; LDL-c, low-density lipoprotein cholesterol; *p< 0.05 for the comparison between NNLH and NASH patients

\begin{tabular}{lccc}
\hline & NNLH (14) & NASH (31) & P \\
\hline Age (years) & $49.2 \pm 6.4$ & $54.8 \pm 7.5$ & 0.21 \\
Male gender (n, \%) & $8(57.1 \%)$ & $18(58.1 \%)$ & - \\
BMI (kg/m²) & $23.2 \pm 1.9$ & $30.5 \pm 3.2$ & $0.001^{*}$ \\
Waist Circumference (cm) & $85.4 \pm 7.4$ & $114 \pm 11.7$ & $0.03^{*}$ \\
ALT (IU/L) & $65.3 \pm 4.6$ & $189.6 \pm 18.1$ & $<0.001^{*}$ \\
AST (IU/L) & $58.4 \pm 6.7$ & $120.2 \pm 10.9$ & $0.01^{*}$ \\
Insulin (ng/mL) & $0.9 \pm 0.2$ & $2.3 \pm 0.4$ & $<0.001^{*}$ \\
Glucose (mmol/L) & $4.8 \pm 1.6$ & $7.9 \pm 3.1$ & 0.13 \\
Triglyceride (mmol/L) & $1.6 \pm 0.2$ & $3.6 \pm 0.4$ & $0.007^{*}$ \\
Total cholesterol (mmol/L) & $5.1 \pm 0.3$ & $4.9 \pm 0.5$ & 0.84 \\
HDL- cholesterol (mmol/L) & $1.4 \pm 0.2$ & $0.8 \pm 0.1$ & $0.001^{*}$ \\
LDL- cholesterol (mmol/L) & $3.5 \pm 0.1$ & $4.9 \pm 0.3$ & $<0.001^{*}$ \\
\hline
\end{tabular}
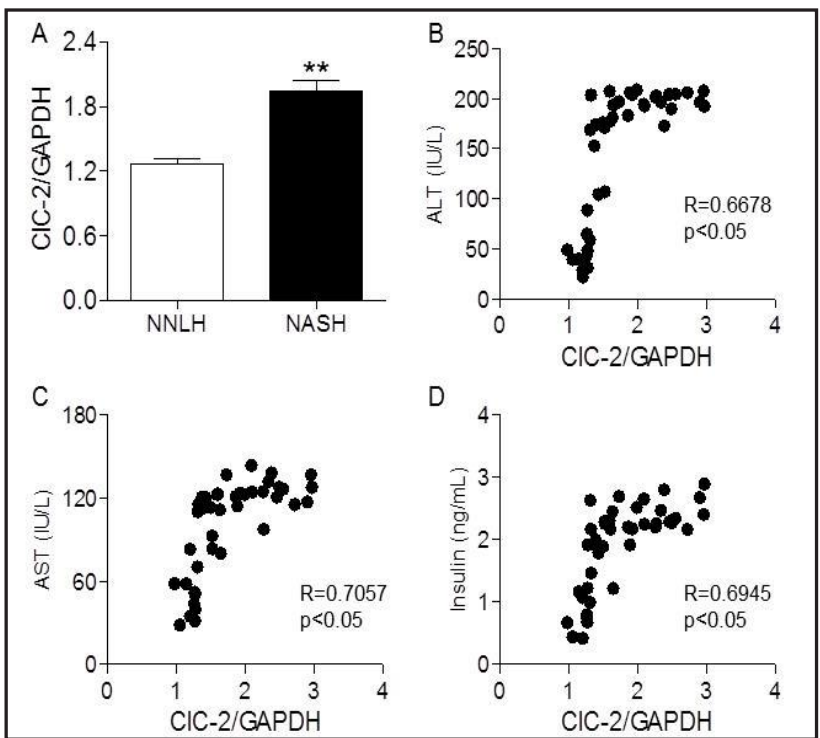

Fig. 1. Increased $\mathrm{ClC}-2$ levels in liver of NASH patients. (A) ClC-2 mRNA level in liver from NNLH or NASH patients. (BD) Correlation between $\mathrm{ClC}-2$ and alanine aminotransferase (ALT) (B), aspartate aminotransferase (AST) (C) or insulin (D) levels. The correlation coefficients were shown, respectively. NNLH patients, $n=14$; NASH patients, $n=31$. 


\section{Effects of CIC-2 inhibition on metabolic parameters and regulators}

To elucidate the mechanism why ClC-2 knockdown inhibits HFD-induced obesity, the whole-body metabolic parameters such as food and water intake, and RER were measured. Although a significant change in food intake was found between chow and HFD treatment, it did not differ before and after ClC-2 inhibition in HFD mice (Fig. 3A). Similarly, water intake remained unchanged among four groups (Fig. 3B). Despite similar food and water intake, AAV-sh-ClC-2 treatment resulted in an increase of RER compared with AAV-null-treated HFD mice (Fig. 3C). Furthermore, the protein expressions of several lipid synthesis-related enzymes in liver were detected by western blotting. The hepatic protein expressions of sterol regulatory element binding protein (SREBP)-1c, fatty acid synthase (FAS), HMG-CoA reductase (HMGCR) and acetyl-Coenzyme A carboxylase (ACC $\alpha$ ) were markedly increased in HFD mice than in chow diet mice. However, knockdown of ClC-2 reversed these changes (Fig. 3D-H).

\section{ClC-2 downregulation ameliorated liver injury}

To investigate the effect of ClC-2 inhibition on hepatic injury during NAFLD, two hepatic enzymes (ALT and AST) that reflect the degree of liver damage were first examined. The results showed that the plasma ALT and AST levels in HFD mice were higher than those of chow diet mice, which was markedly lowered after AAV-sh-ClC-2 treatment (Fig. 4A and B). Consis-

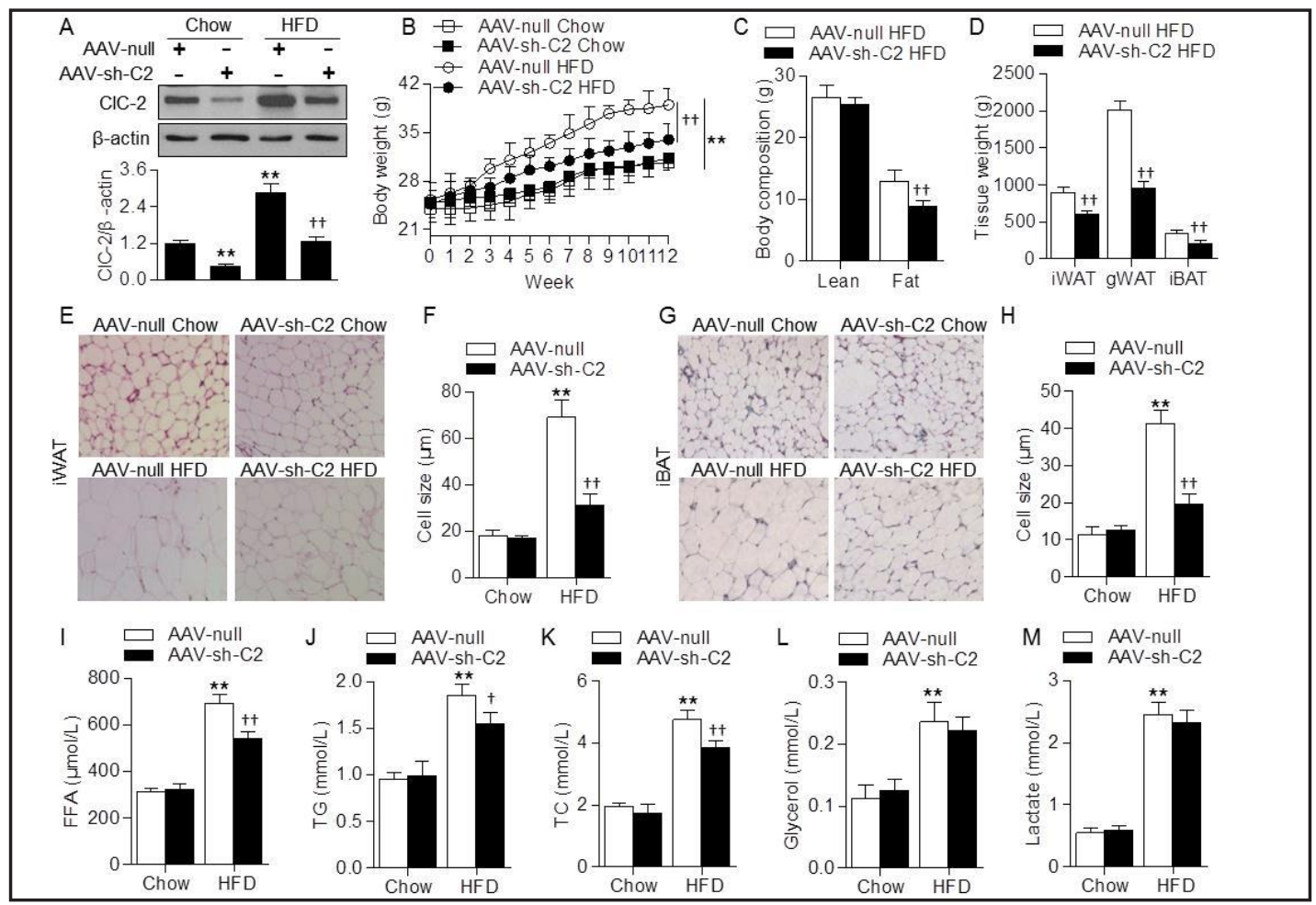

Fig. 2. Inhibition of $\mathrm{ClC}-2$ expression in liver protected from HFD-induced obesity. (A) Starting at 10 weeks of age, mice were injected intravenously with liver specific AAV-sh-ClC-2 (AAV-sh-C2) or AAV-null followed by a chow diet or high fat diet (HFD) for 12 weeks. Western blotting analysis of ClC-2 protein expression in liver ( $n=5$ per group). (B and C) Body weight (B) ( $n=15$ per group) and composition (lean and fat mass) (C) $(n=10$ per group) were measured. (D) Inguinal WAT (iWAT), gonadal WAT (gWAT) and interscapular BAT (iBAT) were analyzed for their weight, respectively. (n=7 per group). (F-H) iWAT (E,F) and iBAT (G, H) were stained with hematoxylin and eosin. The mean diameter of adipocytes was calculated, respectively ( $\mathrm{n}=5$ per group). (I-M) Fasting plasma free fatty acids (FFA) (I), triglyceride (TG) (J), total cholesterol (TC) (K), glycerol (L) and lactate $(\mathrm{M})$ were determined by biochemical assays ( $\mathrm{n}=6$ per group). ${ }^{* *} \mathrm{P}<0.01$ vs. AAV-null Chow; $\uparrow \mathrm{P}<0.05, \uparrow+\mathrm{P}<0.01$ vs AAV-null HFD. 
tent with the changes of plasma lipids, hepatic TC and TG levels were both increased after HFD treatment, while ClC-2 downregulation was associated with decreased hepatic lipids accumulation (Fig. 4C and D). Furthermore, the liver weight and the ratio of liver weight to body weight were higher in HFD mice than in chow diet mice. However, we noticed that AAV-sh-ClC-2-treated mice gained less liver weight than AAV-null-treated mice after HFD feeding (Fig. 4E and F). As displayed in Fig. $4 \mathrm{G}$, no significant differences in liver pathological characteristics were observed between the types of mice fed chow diet. AAV-null treated HFD mice showed marked hepatocyte ballooning and inflammatory cell infiltration, which was significantly attenuated by ClC-2 inhibition. Similar to the effect on TC and TG levels, oil red $\mathrm{O}$ staining revealed that AAVsh-ClC-2 treatment also decreased HFD-induced the deposition of lipid droplets in hepatocytes (Fig. 4H). Additionally, masson trichrome staining showed lower deposition of collagen fibrils in liver in AAV-shClC-2-treated mice than in AAV-null-treated mice after HFD treatment (Fig. 4I).

\section{CIC-2 lacking attenuated HFD-induced insulin resistance}

Glucose metabolism dysfunction and IR are typical clinical symptoms of NAFLD [16]. Mice fed with HFD showed markedly higher fasting blood glucose and insulin. Compared with AAV-null-treated HFD mice, knockdown of ClC-2 displayed reduced blood glucose and insulin (Fig. 5A and B). Furthermore, systemic IR was evaluated using the HOMA-IR index. As shown in Fig. 5C, HFD treatment induced a significant increase of IR, as indicated by the high HOMA-IR value. However, HFD mice lacking ClC-2 showed a marked decrease in HOMA-IR value compared with those of AAV-null-treated HFD mice. GTT and ITT revealed that there were no differences between AAV-null-treated mice and AAV-sh-ClC-2-treated mice on chow diet (Fig. 5D and E). However, on HFD, AAV-sh-ClC-2-treated mice cleared plasma glucose more efficiently after glucose, as well as after insulin intraperitoneal injection (Fig. 5F and G). These results suggest that $\mathrm{ClC}-2$ inhibition can improve glucose clearance and insulin sensitivity.

\section{ClC-2 mediated hepatic insulin signaling pathway}

To assess the underlying mechanisms by which knockdown of ClC-2 prevents IR, the activation of IRS- 1 and downstream pathways were determined. Western blotting showed that AAV-sh-ClC-2-treated HFD mice activated insulin signaling, as evidenced by mildly increased 


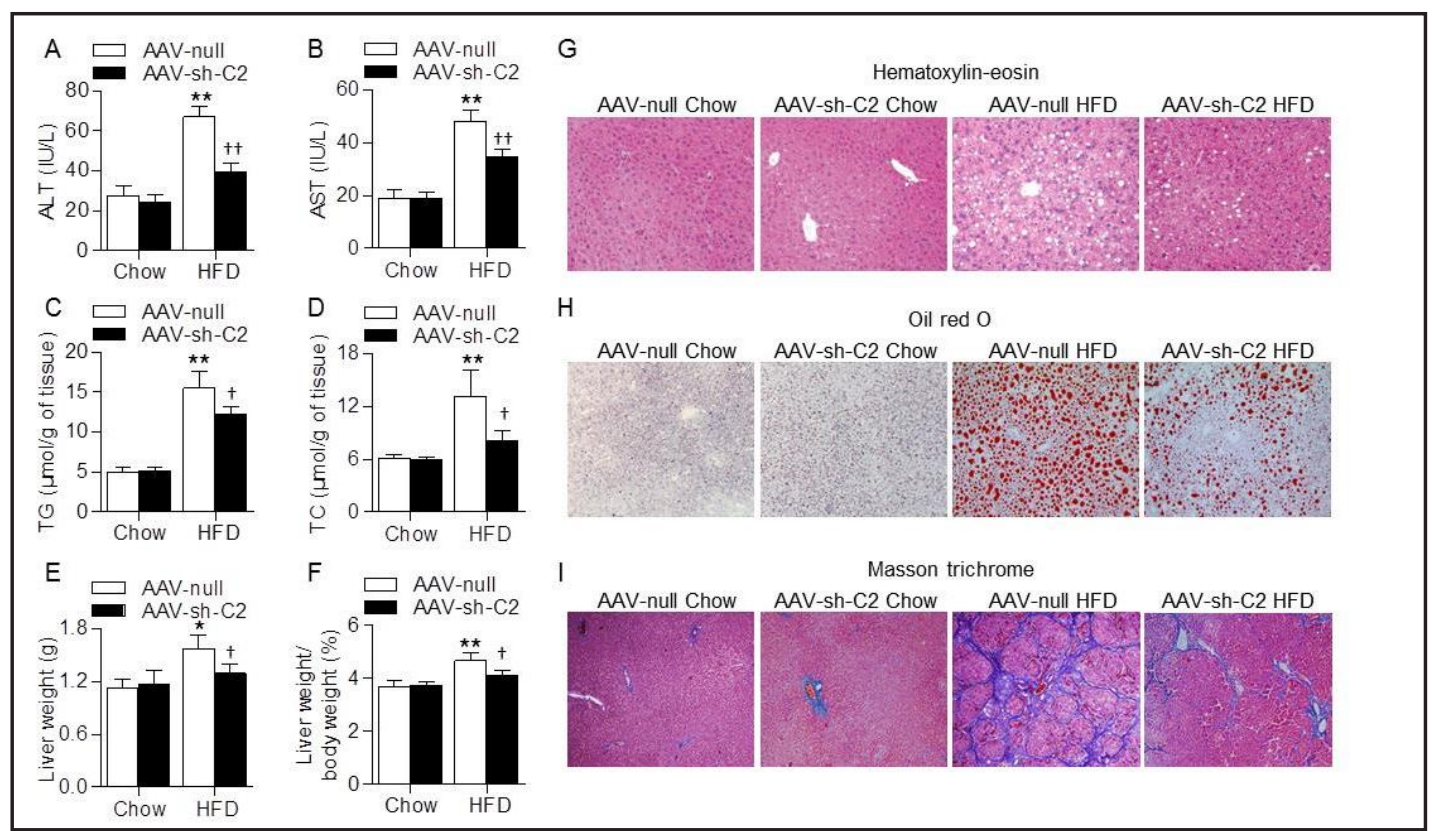

Fig. 4. ClC-2 inhibition ameliorated HFD-induced liver injury. (A-D) Plasma ALT (A), AST (B), TG (C) and TC (D) of AAV-null- or AAV-sh-ClC-2 (AAV-sh-C2)-infected mice on chow diet or HFD for 12 weeks in the fasted state (10 h) ( $\mathrm{n}=10$ per group). ( $\mathrm{E}$ and $\mathrm{F}$ ) Liver weight $(\mathrm{E})$ and the ratio of liver weight to body weight (F) were measured after a 12-week chow diet or HFD (n=8 per group). (G-I) Hematoxylin-eosin (G), oil red $\mathrm{O}(\mathrm{H})$ and masson trichrome (I) staining of liver sections ( $\mathrm{n}=5$ per group). ${ }^{* *} \mathrm{P}<0.01$ vs. AAV-null Chow; $\dagger \mathrm{P}<0.05, \uparrow+\mathrm{P}<0.01$ vs AAV-null HFD.

Fig. 5. ClC-2 lacking attenuated HFDinduced insulin resistance. (A-C) Plasma fasting glucose (A), insulin (B) and homeostasis model assessment of IR (HOMA-IR) value (C) of AAV-null- or AAVsh-ClC-2 (AAV-sh-C2)-infected mice on chow diet or HFD for 12 weeks ( $\mathrm{n}=8$ per group). (D and E) Glucose tolerance test (GTT) (D) and insulin tolerance test (ITT) (E) in AAV-null- or AAV-sh-ClC-2-infected mice on chow diet. (F and G) GTT (F) and ITT (G) were performed after 12 weeks of HFD in AAV-null- or AAV-sh-ClC-2-infected mice ( $\mathrm{n}=12$ per group). ${ }^{* *} \mathrm{P}<0.01$ vs. AAV-null Chow; $\dagger+\mathrm{P}<0.01$ vs AAV-null HFD.

phosphorylation of IRS-1 (Tyr608), Akt (Ser473) and mTOR (Ser2448) and reduced phosphorylation of IRS-1 (Ser307) in liver. Moreover, an acute intraperitoneal injection of insulin dramatically induced insulin signaling activation, which was further enhanced after ClC2 downregulation (Fig. 6A-D). To support the in vivo findings

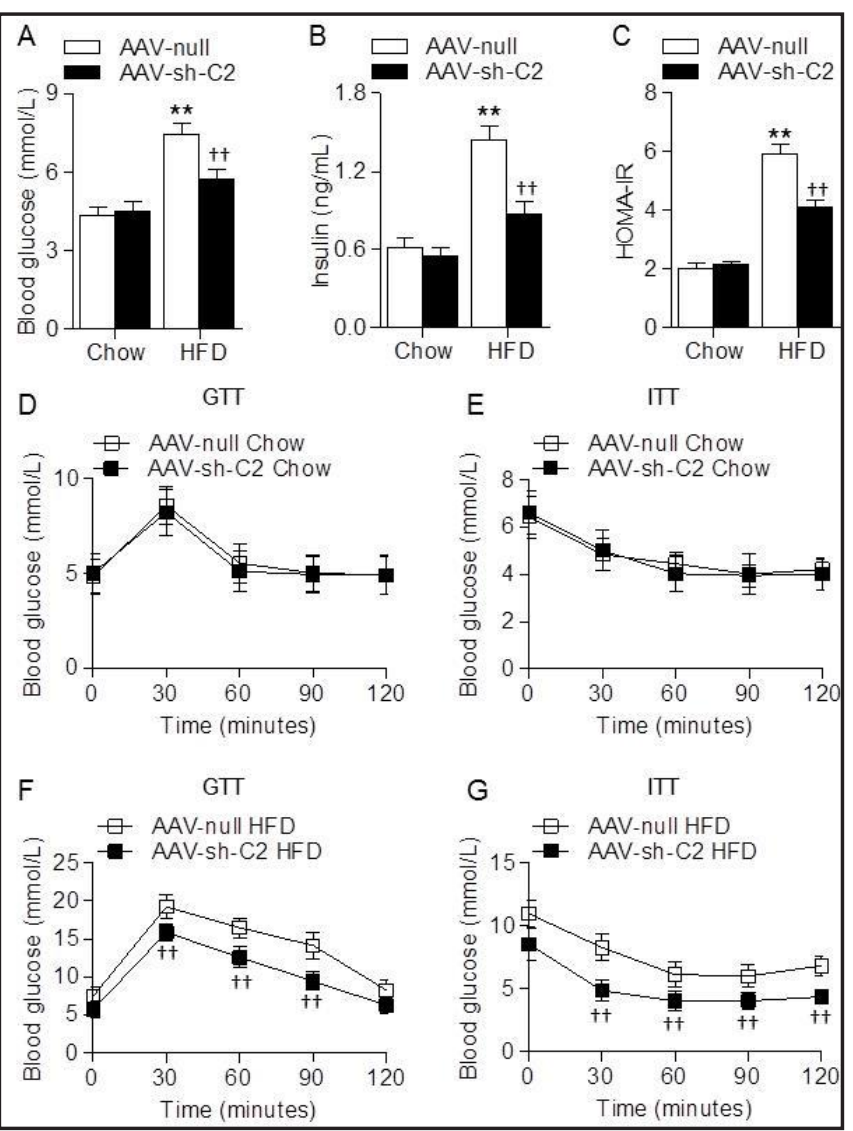




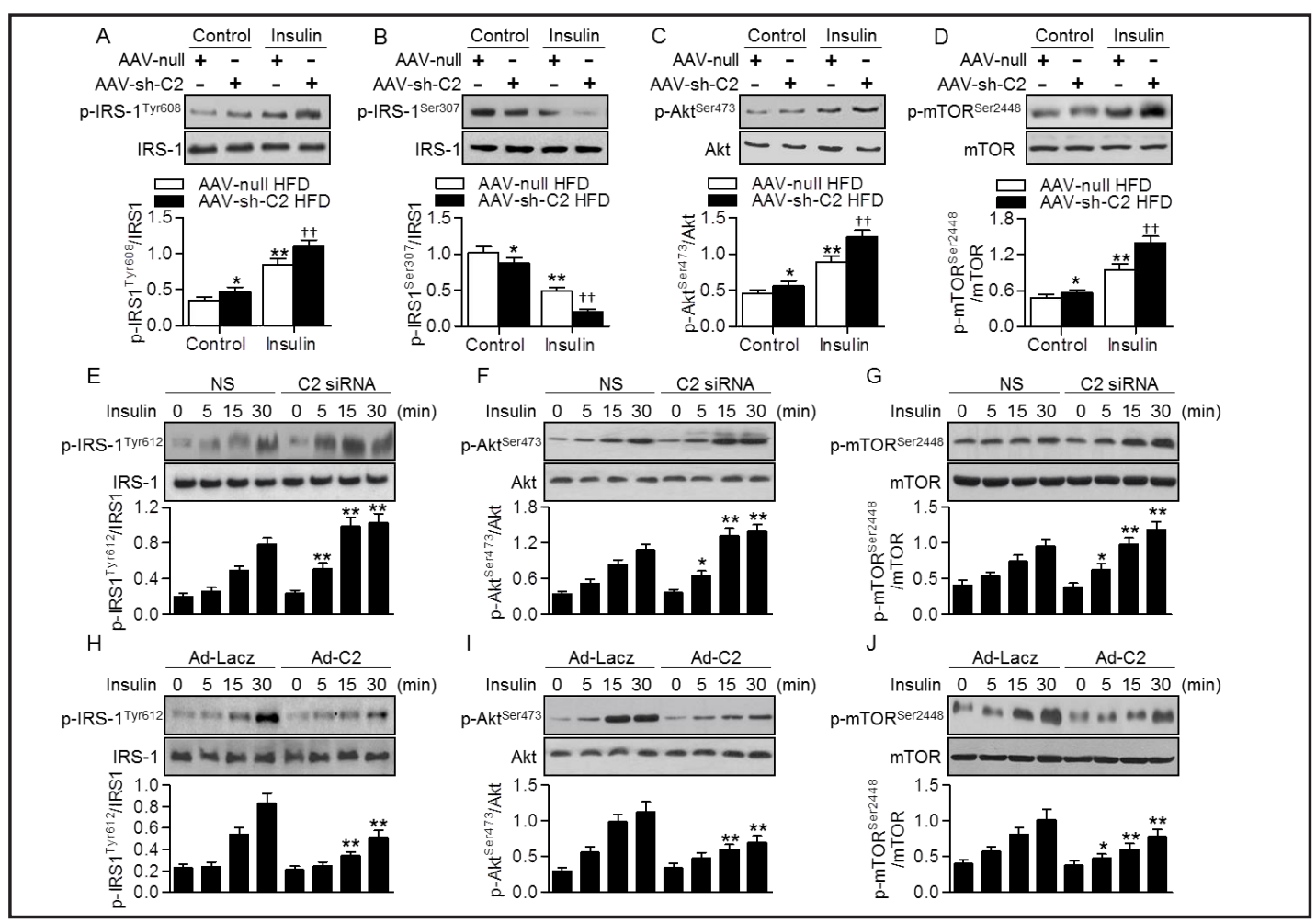

Fig. 6. ClC-2 regulated hepatic insulin signaling pathway. (A-D) phosphorylation of IRS-1 (Tyr608) (A), IRS1(Ser307) (B), Akt (Ser473) (C) and mTOR (Ser2448) (D) under basal conditions (control) and in response to an intraperitoneal injection of insulin $(1.0 \mathrm{U} / \mathrm{kg}$ for $15 \mathrm{~min}$ ) in the liver of AAV-null- or AAV-sh-ClC-2 (AAVsh-C2)-infected mice on HFD ( $\mathrm{n}=12$ per group). ${ }^{*} \mathrm{P}<0.05$, ${ }^{* *} \mathrm{P}<0.01$ vs. AAV-null HFD; $\dagger+\mathrm{P}<0.01$ vs AAV-null HFD+insulin. (E-G) HepG2 cells were treated with ClC-2 siRNA (C2 siRNA) or negative siRNA (NS) for $48 \mathrm{~h}$ before insulin $(100 \mu \mathrm{mol} / \mathrm{L})$ treatment for different times as indicated. Phosphorylation of IRS-1 (Tyr612) (E), Akt (Ser473) (F) and mTOR (Ser2448) (G) were determined (n=6 per group). (H-J) Western blotting analysis of IRS-1 (Tyr612) (H), Akt (Ser473) (I) and mTOR (Ser2448) (J) Phosphorylation in Ad-ClC-2 (AdC2)- or Ad-Lacz-infected HepG2 cells after insulin treatment for different times as indicated (n=8 per group). ${ }^{*} \mathrm{P}<0.05,{ }^{* *} \mathrm{P}<0.01$ vs. corresponding time point in negative siRNA group or Ad-Lacz group.

reported above, human HepG2 cells were exposed to ClC-2 siRNA followed by insulin treatment for different time points as indicated. Our results showed insulin induced phosphorylation of IRS-1 (Tyr608), Akt (Ser473) and mTOR (Ser2448) in negative siRNAtreated cells. These inductions were maintained over a range of indicated time points. Importantly, downregulation of ClC-2 significantly further promoted IRS-1/Akt/mTOR signaling activation (Fig. 6E-G). Although insulin also triggered IRS-1 (Tyr612), Akt (Ser473) and mTOR (Ser2448) phosphorylation in Ad-ClC-2-treated cells, the extent was significantly less than those of Ad-Lacz-treated cells (Fig. 6H-J).

\section{Discussion}

NAFLD has been regarded as a serious public health problem that affects more than $40 \%$ of population in some developed countries [3]. Unfortunately, there is no pharmacological agent currently available for the treatment of NAFLD. It is recently thought that the methods for treating NAFLD include exercise, rational diet and medicines (fibrate, statins and metformin) [2, 5]. However, it still cannot reach consensus on effective drug therapies due to some contraindications and adverse effects [17]. Our present study demonstrates that mice lacking $\mathrm{ClC}-2$ are associated with a remarkably beneficial metabolic phenotype. The results 


\section{Cellular Physiology Cell Physiol Biochem 2018;45:2187-2198

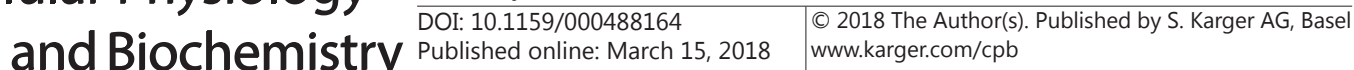

Fu et al.: CIC2 Deficiency Ameliorates Non-Alcoholic Fatty Liver Disease

are summarized as follows: (1) ClC-2 level was significantly increased in liver of NASH patients (sever form of NAFLD) or experimental HFD mice; (2) Clinical data showed positive correlation between hepatic ClC-2 level and plasma ALT, AST or insulin level; (3) Knockdown of hepatic ClC-2 in HFD mice improved the sensitivity to insulin, ameliorated metabolic disorders and liver injury, and limited the development of obesity and liver steatosis. (4) Mechanistically, the anti-IR effect of mice lacking CIC-2 was associated with activation of IRS1 and subsequent Akt/mTOR signaling pathway.

Here, our clinical data demonstrated the critical role of ClC-2 in NAFLD, as evidenced by increased level of ClC-2 in NASH patients compared with NNLH patients. Importantly, the extent of increased ClC-2 expression in liver was positively associated with plasma ALT, AST and insulin levels. Despite the correlation coefficients were not ideal due to relatively small sample amount, they were considered to be statistically significant. Notably, there is a limitation in our present study because of lack of comparison between NASH patient and healthy individuals. In prior to this study, few studies were performed to explore the association between $\mathrm{ClC}-2$ and metabolic diseases. Although a recent study identifying the activation site on ClC-2 induced by fatty acids [15], the functional role of ClC- 2 in metabolic diseases is still unknown. Until now, a study on diabetes mellitus was found. Pan et al. found that high glucose stimulation inhibited keratinocyte migration through decreasing CIC-2 expression, indicating ClC-2 plays a key role during delayed wound healing process [14]. This finding contrasts with our observations in the changes of $\mathrm{ClC}-2$ expressions. The variations in cell types, tissue location and disease state may explain this difference.

Obesity is a major heath issue worldwide and is a complex metabolic syndrome associated with IR and NAFLD [18]. Our study found that HFD mice lacking ClC-2 were resistant to obesity, and leaner than AAV-null-treated HFD mice, which may be resulted from reduced fat mass. Although the food intake was mildly fewer in AAV-sh-ClC-2 HFD mice than in AAV-null HFD mice, it did not fully account for the reduced fat mass observed in AAV-shClC-2 HFD mice. However, RER was significantly elevated after inhibition of ClC-2, indicating the reduced adipose mass may be associated with increased carbohydrate combustion. It has been suggested that high plasma level of FFA contribute to development of NASH [19]. Infusion of TG emulsions to rapidly increase FFA level would result in a reduction of insulin sensitivity [20]. Excessive generation of circulating FFA from WAT can be subsequently absorbed by liver where FFA transforms into TG and bioactive lipid, leading to cause IR and NAFLD $[7,21]$. In this study, the results showed that plasma levels of FFA, TG and TC were elevated in HFD mice, which were inhibited after ClC-2 downregulation. But ClC-2 knockdown produced no effect on the increased levels of glycerol and lactate.

According to the aforementioned 'double-hit' hypothesis, lipid accumulation plays an important role in the 'first hit', whereas the 'second hit' is in combination with oxidative stress and inflammation that eventually leads to liver fibrosis [6]. In the current study, we provided the first evidence that ClC-2 inhibition prevented HFD-induced hepatic lipid accumulation, inflammation and fibrosis. In addition to liver morphology, the hepatic function was also determined, as suggested by the reduced plasma levels of ALT and AST in AAV-sh-ClC-2 HFD mice compared with the AAV-null HFD mice. To investigate the mechanism whereby ClC2 knockdown inhibits lipid accumulation, several lipid metabolism-related enzymes were detected. SREBP-1c is an important transcription factor, regulating lipid synthesis and fatty acid metabolism in liver [22]. Moreover, SREBP-1c also regulates several genes involved in the de novo pathway, such as FAS and ACC $\alpha$, mediating hepatic synthesis of fatty acids, TG and cholesterol [23]. In the way of cholesterol metabolism, HMGCR is a crucial enzyme in the synthesis process of cholesterol [23]. Our results found that HFD mice lacking ClC-2 showed suppressed de novo lipid and cholesterol synthesis in the liver by inhibiting the protein expressions of SREBP-1c, FAS, ACC $\alpha$ and HMGCR.

Our data also demonstrated that knockdown of ClC-2 in HFD mice decreased HOMAIR value and attenuated whole-body glucose intolerance and IR. IR, besides hepatic lipid accumulation in the 'first hit', is also the first step towards NAFLD [6]. Insulin functions as an important role in regulating hepatic glycogen synthesis and glucose homeostasis 


\section{Cellular Physiology Cell Physiol Biochem 2018;45:2187-2198 \begin{tabular}{l|l|l} 
DOI: 10.1159/000488164 & and Biochemistry Published online: March 15, 2018 & $\begin{array}{l}\text { O 2018 The Author(s). Published by S. Karger AG, Basel } \\
\text { www.karger.com/cpb }\end{array}$
\end{tabular}

[16]. IRS-1 is the major insulin receptor effector that responsible for the transduction of insulin signaling. Upon the binding of insulin to insulin receptor, IRS-1 is phosphorylated at Tyr608/612, leading to insulin pathway activation $[16,24]$. On the contrary, phosphorylation of IRS-1 at Ser307/312 is considered a negative feedback marker that blocks insulin signaling, causing glucose uptake impairment and IR [25]. Furthermore, the Akt pathway is thought to be a critical component of insulin signaling cascade, and it is mainly activated at Ser473 [18]. Phosphorylation of Akt can stimulate mTOR by phosphorylating it at Ser2448. Multiple evidences have suggested that dysregulation of the Akt/mTOR signaling pathway is the major molecular mechanism underlying the metabolic dysfunctions such as NAFLD [26]. In the present study, we showed that hepatic ClC-2 deficiency in HFD mice enhanced phosphorylation of IRS-1 at Tyr608 as well as further inhibited Ser307 phosphorylation induced by acute intraperitoneal injection of insulin. Additionally, inhibition of ClC-2 expression in HepG2 cells promoted insulin-induced the activation of IRS-1/Akt/mTOR signaling pathway, whereas ClC-2 overexpression exerted the opposite effects.

\section{Conclusion}

Our study has revealed a critical involvement of ClC- 2 in regulating metabolic disorders. Downregulation of ClC-2 alleviates HFD-induced obesity, hepatic lipid accumulation, glucose intolerance and IR via strengthening IRS-1/Akt/mTOR signaling pathway. These findings indicate that inhibition of ClC-2 may be a potentially powerful therapeutic strategy for the patients with NAFLD.

\section{Abbreviations}

NAFLD (non-alcoholic fatty liver disease); NASH (non-alcoholic steatohepatitis); ClC-2 (chloride channel 2); HFD (high fat diet); AAV (adeno-associated virus); IR (insulin resistance); FFA (free fatty acid); TG (triglyceride); TC (total cholesterol); ALT (alanine transaminase); AST (aspartate transaminase); WAT (white adipose tissue); BAT (brown adipose tissue); HOMA-IR (homeostasis model of insulin resistance); RER (respiratory exchange ratio); SREBP-1c (sterol regulatory element binding protein-1c); FAS (fatty acid synthase); HMGCR (HMG-CoA reductase); ACC $\alpha$ (acetyl-Coenzyme A carboxylase); GTT (glucose tolerance test); ITT (insulin tolerance test); IRS-1 (insulin receptor substrates 1)

\section{Disclosure Statement}

The authors declare that they have no conflict of interest

\section{References}

$>1$ Wree A, Broderick L, Canbay A, Hoffman HM, Feldstein AE: From NAFLD to NASH to cirrhosis-new insights into disease mechanisms. Nat Rev Gastroenterol Hepatol 2013;10:627-636.

-2 Neuschwander-Tetri BA, Clark JM, Bass NM, Van Natta ML, Unalp-Arida A, Tonascia J, Zein CO, Brunt EM, Kleiner DE, McCullough AJ, Sanyal AJ, Diehl AM, Lavine JE, Chalasani N, Kowdley KV, Network NCR: Clinical, laboratory and histological associations in adults with nonalcoholic fatty liver disease. Hepatology 2010;52:913-924.

3 Mulhall BP, Ong JP, Younossi ZM: Non-alcoholic fatty liver disease: an overview. J Gastroenterol Hepatol 2002;17:1136-1143.

4 Anstee QM, Targher G, Day CP: Progression of NAFLD to diabetes mellitus, cardiovascular disease or cirrhosis. Nat Rev Gastroenterol Hepatol 2013;10:330-344. 


\section{Cellular Physiology Cell Physiol Biochem 2018;45:2187-2198 \begin{tabular}{ll|l} 
DOI: 10.1159/000488164 & O 2018 The Author(s). Published by S. Karger AG, Basel \\
www.karger.com/cpb
\end{tabular}}

Fu et al.: CIC2 Deficiency Ameliorates Non-Alcoholic Fatty Liver Disease

5 Harrison SA, Day CP: Benefits of lifestyle modification in NAFLD. Gut 2007;56:1760-1769.

6 Day CP, James OF: Steatohepatitis: a tale of two "hits"? Gastroenterology 1998;114:842-845.

7 Utzschneider KM, Kahn SE: Review: The role of insulin resistance in nonalcoholic fatty liver disease. J Clin Endocrinol Metab 2006;91:4753-4761.

8 Accardi A: Structure and gating of CLC channels and exchangers. J Physiol 2015;593:4129-4138.

-9 McCallum L, Jeemon P, Hastie CE, Patel RK, Williamson C, Redzuan AM, Dawson J, Sloan W, Muir S, Morrison D, McInnes GT, Freel EM, Walters M, Dominiczak AF, Sattar N, Padmanabhan S: Serum chloride is an independent predictor of mortality in hypertensive patients. Hypertension 2013;62:836-843.

-10 Yang H, Huang LY, Zeng DY, Huang EW, Liang SJ, Tang YB, Su YX, Tao J, Shang F, Wu QQ, Xiong LX, Lv XF, Liu J, Guan YY, Zhou JG: Decrease of intracellular chloride concentration promotes endothelial cell inflammation by activating nuclear factor-kappaB pathway. Hypertension 2012;60:1287-1293.

11 Wu QQ, Liu XY, Xiong LX, Shang JY, Mai XY, Pang RP, Su YX, Yu BX, Yuan JN, Yang C, Wang YL, Zhou P, Lv XF, Liu J, Zhou JG, Liang SJ: Reduction of Intracellular Chloride Concentration Promotes Foam Cell Formation. Circ J 2016;80:1024-1033.

12 Wang H, Xu M, Kong Q Sun P, Yan F, Tian W, Wang X: Research and progress on ClC2 (Review). Mol Med Rep 2017;16:11-22.

13 Roman RM, Smith RL, Feranchak AP, Clayton GH, Doctor RB, Fitz JG: ClC-2 chloride channels contribute to HTC cell volume homeostasis. Am J Physiol Gastrointest Liver Physiol 2001;280:G344-353.

14 Pan F, Guo R, Cheng W, Chai L, Wang W, Cao C, Li S: High glucose inhibits ClC-2 chloride channels and attenuates cell migration of rat keratinocytes. Drug Des Devel Ther 2015;9:4779-4791.

15 Cuppoletti J, Tewari KP, Chakrabarti J, Malinowska DH: Identification of the fatty acid activation site on human ClC-2 Am J Physiol Cell Physiol 2017;312:C707-C723.

16 Kumashiro N, Erion DM, Zhang D, Kahn M, Beddow SA, Chu X, Still CD, Gerhard GS, Han X, Dziura J, Petersen KF, Samuel VT, Shulman GI: Cellular mechanism of insulin resistance in nonalcoholic fatty liver disease. Proc Natl Acad Sci U S A 2011;108:16381-16385.

17 Smith BW, Adams LA: Non-alcoholic fatty liver disease. Crit Rev Clin Lab Sci 2011;48:97-113.

18 Czech MP: Insulin action and resistance in obesity and type 2 diabetes. Nat Med 2017;23:804-814.

19 Csak T, Ganz M, Pespisa J, Kodys K, Dolganiuc A, Szabo G: Fatty acid and endotoxin activate inflammasomes in mouse hepatocytes that release danger signals to stimulate immune cells. Hepatology 2011;54:133-144.

20 Staehr P, Hother-Nielsen O, Landau BR, Chandramouli V, Holst JJ, Beck-Nielsen H: Effects of free fatty acids per se on glucose production, gluconeogenesis, and glycogenolysis. Diabetes 2003;52:260-267.

21 Samuel VT, Shulman GI: Mechanisms for insulin resistance: common threads and missing links. Cell 2012;148:852-871.

22 Ahmed MH, Byrne CD: Modulation of sterol regulatory element binding proteins (SREBPs) as potential treatments for non-alcoholic fatty liver disease (NAFLD). Drug Discov Today 2007;12:740-747.

-23 Ameer F, Scandiuzzi L, Hasnain S, Kalbacher H, Zaidi N: De novo lipogenesis in health and disease. Metabolism 2014;63:895-902.

-24 Taniguchi CM, Ueki K, Kahn R: Complementary roles of IRS-1 and IRS-2 in the hepatic regulation of metabolism. J Clin Invest 2005;115:718-727.

-25 Thirone AC, Huang C, Klip A: Tissue-specific roles of IRS proteins in insulin signaling and glucose transport. Trends Endocrinol Metab 2006;17:72-78.

-26 Hwang HJ, Jung TW, Kim BH, Hong HC, Seo JA, Kim SG, Kim NH, Choi KM, Choi DS, Baik SH, Yoo HJ: A dipeptidyl peptidase-IV inhibitor improves hepatic steatosis and insulin resistance by AMPK-dependent and JNK-dependent inhibition of LECT2 expression. Biochem Pharmacol 2015;98:157-166. 